Magdalena Nockowska-Rosiak (Łódź)

\title{
ECONOMIC EQUILIBRIUM THROUGH VARIATIONAL INEQUALITIES
}

Abstract. The purpose of this paper is to present an alternative proof of the existence of the Walrasian equilibrium for the Arrow-Debreu-McKenzie model by the variational inequality technique. Moreover, examples of the generalized Arrow-Debreu-McKenzie model are given in which the price vector can reach the boundary of the orthant allowing a commodity to be of price zero at equilibrium. In such a case its supply exceeds demand. It is worth mentioning that utility functions in this model are allowed not to be strictly concave and they can reach their maximum in the commodity space.

1. Introduction. Let us consider the Arrow-Debreu-McKenzie model with $m$ consumers (indexed by $j \in J:=\{1, \ldots, m\}$ ), $n$ firms (indexed by $i \in I:=\{1, \ldots, n\}$ ), and $s$ goods (indexed by $l \in L:=\{1, \ldots, s\}$ ). In such economy, society's initial endowments and technological possibilities (i.e., the firms) are owned by consumers. The preference of the $j$ th consumer is represented by a utility function, denoted by $u_{j}$. The initial endowment of the $j$ th consumer is given by $\omega_{j} \in \mathbb{R}_{+}^{n}$. In addition, we suppose that consumer $j$ owns a share $\kappa_{j i}$ of firm $i$, where $\sum_{j \in J} \kappa_{j i}=1$. Denote by $Y_{i} \subset \mathbb{R}^{n}$ the production set associated with the $i$ th firm.

Recall that an allocation $\left(x_{1}^{\star}, \ldots, x_{m}^{\star}, y_{1}^{\star}, \ldots, y_{n}^{\star}\right), x_{j}^{\star} \in \mathbb{R}_{+}^{n}, j \in J, y_{i}^{\star} \in$ $\mathbb{R}^{n}, i \in I$, and price vector $\pi \in \operatorname{Int} \mathbb{R}_{+}^{n}$ constitute a classical competitive (or Walrasian) equilibrium if the following conditions are satisfied ([6]):

- Profit maximization: For each firm $i \in I, y_{i}^{\star}$ solves

$$
\max _{y_{i} \in Y_{i}}\left\langle\pi, y_{i}\right\rangle
$$

2000 Mathematics Subject Classification: Primary 91B50; Secondary 65K10.

Key words and phrases: optimization problem, Walrasian equilibrium, variational inequalities. 
- Utility maximization: For each consumer $j \in J, x_{j}^{\star}$ solves

$$
\max \left\{u_{j}\left(x_{j}\right):\left\langle\pi, x_{j}\right\rangle \leq\left\langle\pi, \omega_{j}\right\rangle+\sum_{i \in I} \kappa_{i j}\left\langle\pi, y_{i}^{\star}\right\rangle, x_{j} \in \mathbb{R}_{+}^{n}\right\} ;
$$

- Market clearing:

$$
\sum_{j \in J} x_{j}^{\star}=\sum_{j \in J} \omega_{j}+\sum_{i \in I} y_{i}^{\star} .
$$

If $Y_{i}=\{0\}$ for all $i \in J$, then the Arrow-Debreu-McKenzie model is the Arrow-Debreu model of pure exchange (cf. [1], [2], [5], [12]). [12]).

It is usually assumed that $\pi \in \operatorname{Int} \mathbb{R}_{+}^{n}$ or $\pi \in \operatorname{Fr} \mathbb{R}_{+}^{n}, \pi \neq 0$ (cf. [1], [5],

For the Arrow-Debreu-McKenzie model we introduce the functions

$$
\begin{gathered}
V_{j}:=-u_{j}, \quad \phi_{j}(\pi):=\left\langle\pi, \omega_{j}\right\rangle+\sum_{i \in I} \kappa_{i j} \sup _{y_{i} \in Y_{i}}\left\langle\pi, y_{i}\right\rangle, \quad j=1, \ldots, m, \\
\Phi(\pi):=\sum_{j \in J} \phi_{j}(\pi)=\left\langle\pi, \sum_{j \in J} \omega_{j}\right\rangle+\sum_{i \in I} \sup _{y_{i} \in Y_{i}}\left\langle\pi, y_{i}\right\rangle, \quad \pi \in \mathbb{R}_{+}^{n} .
\end{gathered}
$$

For the Arrow-Debreu model we set $V_{j}:=-u_{j}, \phi_{j}(\pi):=\left\langle\pi, \omega_{j}\right\rangle, j=$ $1, \ldots, m$, and $\Phi(\pi):=\sum_{j \in J} \phi_{j}(\pi)=\left\langle\pi, \sum_{j \in J} \omega_{j}\right\rangle$.

In both models instead of (1.1) the following variational inequality, called the balance condition, will be considered:

$$
\left\langle\tau-\pi,-\sum_{j \in J} x_{j}^{\star}\right\rangle+\Phi(\tau)-\Phi(\pi) \geq 0, \quad \forall \tau \in \mathbb{R}_{+}^{n} .
$$

It states that the market clears for a commodity if its equilibrium price is positive. Otherwise, there may be an excess supply of the commodity in equilibrium and then its price is zero ([5], [12]).

Thus, we get the following problem: Find $\pi \in \mathbb{R}_{+}^{n}, \pi \neq 0$, and $x_{j} \in \mathbb{R}_{+}^{n}$, $j=1, \ldots, m$, such that

$$
\left\{\begin{array}{l}
V_{j}\left(x_{j}\right)=\min \left\{V_{j}(x):\langle\pi, x\rangle \leq \phi_{j}(\pi), x \in \mathbb{R}_{+}^{n}\right\}, \\
\left\langle-\sum_{j=1}^{m} x_{j}, \tau-\pi\right\rangle+\Phi(\tau)-\Phi(\pi) \geq 0, \quad \forall \tau \in \mathbb{R}_{+}^{n} .
\end{array}\right.
$$

The problem $(P)$ has been first considered under the assumption that $\phi_{j}(\pi)$ $\geq \gamma_{j}$ for some $\gamma_{j}>0$ (cf. [7]). In further research the functions $\phi_{j}$ were assumed to be nonnegative, continuous, positive homogeneous of degree 1 and $\Phi=\sum_{j=1}^{m} \phi_{j}$ (see [8]-[10]).

The results of [8], [9] allow us to prove the existence of a classical competitive (Walrasian) equilibrium in the alternative way in Section 3.

The results in [10] include examples with classical assumptions while for certain cases the assumption of strict monotonicity of $-V_{j}$ is substantially 
weakened. Rejection of this assumption leads to a significant consequence: the problem $(P)$ can be replaced by the problem $(P Q)$ : Find $r \in(0,1]$, $\pi \in \mathbb{R}_{+}^{n}, \pi \neq 0$, and $x_{j} \in \mathbb{R}_{+}^{n}, \alpha_{j} \in \mathbb{R} \cup\{\infty\}, j=1, \ldots, m$, such that

$$
\left\{\begin{array}{l}
-\alpha_{j} \pi \in \partial \bar{V}_{j}\left(x_{j}\right),\left\langle\pi, x_{j}\right\rangle-\phi_{j}(\pi) \in \partial \operatorname{ind}_{\geq 0}\left(\alpha_{j}\right), \quad \text { if } \alpha_{j} \in \mathbb{R}_{+}, \\
-\pi \in \partial^{\infty} \bar{V}_{j}\left(x_{j}\right), \quad\left\langle\pi, x_{j}\right\rangle=\phi_{j}(\pi)=0, \quad \text { if } \alpha_{j}=\infty, \\
\Phi(\tau)-\Phi(\pi) \geq\left\langle\tau-\pi, \frac{1}{r} \sum_{j=1}^{m} x_{j}\right\rangle, \quad \forall \tau \in \mathbb{R}_{+}^{n} .
\end{array}\right.
$$

Notice that if $r=1$ then a solution of the problem $(P Q)$ becomes a Walrasian equilibrium in the sense of the Arrow-Debreu model with $\pi \in \mathbb{R}_{+}^{n}$ (in the classical approach, $\pi \in \operatorname{Int} \mathbb{R}_{+}^{n}$ ). The case of $\alpha_{j}=\infty$ does not imply that $x_{j}$ is a maximizer of the utility function $-V_{j}$ on the budget set $\{x:\langle\pi, x\rangle=0$, $\left.x \in \mathbb{R}_{+}^{n}\right\}$. Such a bundle can be regarded worthless for the $j$ th trader, meaning that he cannot gain any commodities with a positive price. Any traders with zero initial endowment are obviously irrelevant to the market. A solution of the problem $(P Q)$ with $r \in(0,1)$ describes the situation when the total supply exceeds the total demand on the market; such a solution cannot be regarded as a Walrasian equilibrium in the classical sense. A solution of the problem $(P Q)$ with $r \in(0,1)$ can occur only when for at least one trader the value of his commodity bundle does not reach the budget line.

Conditions guaranteeing that $r=1$ and $\pi \in \operatorname{Int} \mathbb{R}_{+}^{n}$ are given in Section 4 . In Section 4 three markets with two traders and two commodities are considered. In the second example the solution of the problem $(P Q)$ is the Walrasian equilibrium. In the first example both coordinates of the price vectors obtained are positive. In the remaining ones, the second coordinate of the price vectors is 0 . In each example, the price vector is unique on the sphere. Moreover, in all examples at least one utility function attains its global maximum on $\mathbb{R}_{+}^{2}$.

2. Statement of the problem and preliminaries. Denote by $\mathbb{R}^{n}$ the Euclidean vector space equipped with the inner product $\langle\cdot, \cdot\rangle: \mathbb{R}^{n} \times \mathbb{R}^{n} \rightarrow \mathbb{R}$. Set

$$
\mathbb{R}_{+}^{n}=\left\{x=\left[x_{1}, \ldots, x_{n}\right] \in \mathbb{R}^{n}: x_{i} \geq 0, \forall i=1, \ldots, n\right\} .
$$

Denote by $\operatorname{ind}_{K}$ the indicator function of a set $K$, i.e.

$$
\operatorname{ind}_{K}(y)= \begin{cases}0 & \text { if } y \in K \\ \infty & \text { otherwise. }\end{cases}
$$

Throughout the paper it will be assumed that the functions

$$
V_{j}: \mathbb{R}^{n} \rightarrow \mathbb{R} \cup\{\infty\}, \quad j=1, \ldots, m,
$$

are convex, proper and lower semicontinuous, and we set $\bar{V}_{j}:=V_{j}+\operatorname{ind}_{\mathbb{R}_{+}^{n}}$. 
Assume that the functions

$$
\phi_{j}: \mathbb{R}^{n} \rightarrow \mathbb{R}_{+}, \quad \phi_{j} \not \equiv 0, \quad j=1, \ldots, m,
$$

are continuous and positive homogeneous of degree 1, i.e.

$$
\phi_{j}(t \tau)=t \phi_{j}(\tau), \quad \forall \tau \in \mathbb{R}_{+}^{n}, \forall t>0 .
$$

Furthermore, let

$$
\Phi: \mathbb{R}^{n} \rightarrow \mathbb{R} \cup\{\infty\}, \quad \Phi=\sum_{j=1}^{m} \phi_{j} ;
$$

it is a convex, proper, lower semicontinuous function, positive homogeneous of degree 1.

We consider the problem $(P)$ : Find $\pi \in \mathbb{R}_{+}^{n}, \pi \neq 0$, and $x_{j} \in \mathbb{R}_{+}^{n}$, $j=1, \ldots, m$, such that

$$
\left\{\begin{array}{l}
V_{j}\left(x_{j}\right)=\min \left\{V_{j}(x):\langle\pi, x\rangle \leq \phi_{j}(\pi), x \in \mathbb{R}_{+}^{n}\right\}, \\
\left\langle-\sum_{j=1}^{m} x_{j}, \tau-\pi\right\rangle+\Phi(\tau)-\Phi(\pi) \geq 0, \quad \tau \in \mathbb{R}_{+}^{n} .
\end{array}\right.
$$

REMARK 2.1. The problem $(P)$ has an equivalent form: find $\left(\pi,\left(x_{j}\right),\left(\alpha_{j}\right)\right)$ $\in \mathbb{R}_{+}^{n} \times\left(\mathbb{R}_{+}^{n}\right)^{m} \times\left(\mathbb{R}_{+}\right)^{m}$ such that

$$
\left\{\begin{array}{l}
-\alpha_{j} \pi \in \partial \bar{V}_{j}\left(x_{j}\right), \\
\left\langle\pi, x_{j}\right\rangle-\phi_{j}(\pi) \in \partial \operatorname{ind}_{\geq 0}\left(\alpha_{j}\right), \\
\Phi(\tau)-\Phi(\pi) \geq\left\langle\tau-\pi, \sum_{j=1}^{m} x_{j}\right\rangle, \quad \forall \tau \in \mathbb{R}_{+}^{n},
\end{array}\right.
$$

where $\left(\alpha_{j}\right)$ are the Lagrange multipliers for the problem $(P)$.

REMARK 2.2. Recall that if $H$ is a Hilbert space and $V: H \rightarrow \mathbb{R} \cup\{\infty\}$ is a convex function, the subdifferential $\partial V: H \rightarrow 2^{H}$ is defined by

$$
\partial V(u)=\{w \in H: V(v)-V(u) \geq\langle w, v-u\rangle, \forall v \in H\}
$$

provided that $V(u)<\infty$, and $\partial V(u)=\emptyset$ otherwise.

Moreover, for a convex set $K$ and $x \in K, \partial \operatorname{ind}_{K}(x)$ is called the normal cone to $K$ at $x$ and is denoted by $N_{K}(x)$ (see [3]). For a convex function $V: X \rightarrow \mathbb{R} \cup\{\infty\}, X$ being a Banach space, the asymptotic generalized gradient of $V$ at $x$ is defined by

$$
\partial^{\infty} V(x):=\left\{x^{\star} \in X^{\star}:\left(x^{\star}, 0\right) \in N_{\text {epi } V}(x, V(x))\right\},
$$

where epi $V$ denotes the epigraph of $V$ (see [4], [11]).

REMARK 2.3. For the function $\Phi_{+}=\Phi+\operatorname{ind}_{\mathbb{R}_{+}^{n}}$, which is convex, lower semicontinuous, and positive homogeneous of degree 1, there exists a nonempty, convex, closed set $W \subset \mathbb{R}^{n}$ such that $\Phi_{+}(\tau)=\sup _{y \in W}\langle\tau, y\rangle$ for $\tau \in \mathbb{R}_{+}^{n}$ (see [3]). 
3. The existence of a competitive equilibrium. In the classical approach for the Arrow-Debreu-McKenzie and Arrow-Debreu models the vector price is positive and it is assumed that the function $V_{j}=-u_{j}$ satisfies one of the conditions:

$\left(H_{3}^{0}\right) V_{j}$ is strictly decreasing on $\mathbb{R}_{+}^{n}$, that is, $V_{j}(x+y)<V_{j}(x)$ for all $x \in \mathbb{R}_{+}^{n}$ and $y \in \mathbb{R}_{+}^{n} \backslash\{0\}$,

or

$\left(H_{3}^{1}\right) V_{j}$ is strictly decreasing on Int $\mathbb{R}_{+}^{n}$, that is, $V_{j}(x+y)<V_{j}(x)$ for all $x \in \operatorname{Int} \mathbb{R}_{+}^{n}$ and $y \in \mathbb{R}_{+}^{n} \backslash\{0\}$, and moreover $V_{j}(x)<V_{j}(y)$ for all $x \in \operatorname{Int} \mathbb{R}_{+}^{n}$ and $y \in \operatorname{Fr} \mathbb{R}_{+}^{n}$.

The existence of a competitive equilibrium for the Arrow-Debreu-McKenzie and Arrow-Debreu models follows from the results in [8] for the case $A_{j}=I$, the identity matrix.

ThEOREM 3.1 ([8, Theorem 3, p. 66]). Suppose that for any $j=1, \ldots, m$ the following conditions are satisfied:

$\left(H_{1}\right) \quad 0 \in \operatorname{cl}\left(\operatorname{Dom} \partial \bar{V}_{j}\right)$ and $\left(\mathbb{R}_{-}^{n} \backslash\{0\}\right) \cap B_{\mathbb{R}^{n}}\left(0, r_{j}\right) \subset \operatorname{Int} \operatorname{Dom} \bar{V}_{j}^{\star}$ for some $r_{j}>0$

$\left(H_{2}\right)\left\{x \in \mathbb{R}_{+}^{n}:\left\{\left\langle x^{\star}, x\right\rangle: x^{\star} \in \partial \bar{V}_{j}(x)\right\} \cap \mathbb{R}_{-} \neq \emptyset\right\} \subset B_{\mathbb{R}^{n}}\left(0, M_{j}\right)$ for some $M_{j}>0$

$\left(H_{4}\right) \gamma_{j}:=\min \left\{\phi_{j}(\tau): \tau \in \mathbb{R}_{+}^{n},|\tau|=1\right\}>0$

$\left(H_{5}\right) \quad \sum_{j=1}^{m} x_{j} \notin \partial \Phi_{+}(0)$ for any $x_{j} \in \partial \bar{V}_{j}^{\star}(0)$.

Then there exist $s \geq 1$ and $\left(\pi,\left(x_{j}\right),\left(\alpha_{j}\right)\right) \in \mathbb{R}_{+}^{n} \times\left(\mathbb{R}_{+}^{n}\right)^{m} \times\left(\mathbb{R}_{+}\right)^{m}, \pi \neq 0$, such that

$$
\left\{\begin{array}{l}
-\alpha_{j} \pi \in \partial \bar{V}_{j}\left(x_{j}\right), \\
\left\langle\pi, x_{j}\right\rangle-s \phi_{j}(\pi) \in \partial \operatorname{ind}_{\geq 0}\left(\alpha_{j}\right), \\
\Phi(\tau)-\Phi(\pi) \geq\left\langle\tau-\pi, \sum_{j=1}^{m} x_{j}\right\rangle, \quad \forall \tau \in \mathbb{R}_{+}^{n} .
\end{array}\right.
$$

Now we are in a position to prove the following theorem:

THEOREM 3.2. Suppose that for any $j=1, \ldots, m$ the following conditions are satisfied:

$\left(H_{1}\right) \quad 0 \in \operatorname{cl}\left(\operatorname{Dom} \partial \bar{V}_{j}\right)$ and $\left(\mathbb{R}_{-}^{n} \backslash\{0\}\right) \cap B_{\mathbb{R}^{n}}\left(0, r_{j}\right) \subset \operatorname{Int} \operatorname{Dom} \bar{V}_{j}^{\star}$ for some $r_{j}>0$

$\left(H_{6}\right) \phi_{j}(\tau)>0$ for all $\tau \in \operatorname{Int} \mathbb{R}_{+}^{n}$;

$\left(H_{7}\right)$ there exists $\delta>0$ such that $\delta\|\tau\| \geq \Phi(\tau)$ for all $\tau \in \mathbb{R}_{+}^{n}$.

Moreover assume that for any $j=1, \ldots, m$ condition $\left(H_{3}^{0}\right)$ or $\left(H_{3}^{1}\right)$ holds. Then there exists a solution of the following problem $(\widehat{P})$ : Find $\pi \in \operatorname{Int} \mathbb{R}_{+}^{n}$ 
and $x_{j} \in \mathbb{R}_{+}^{n}, j=1, \ldots, m$, such that

$$
\left\{\begin{array}{l}
V_{j}\left(x_{j}\right)=\min \left\{V_{j}(x):\langle\pi, x\rangle \leq \phi_{j}(\pi), x \in \mathbb{R}_{+}^{n}\right\}, \quad j=1, \ldots, m, \\
\left\langle-\sum_{j=1}^{m} x_{j}, \tau-\pi\right\rangle+\Phi(\tau)-\Phi(\pi) \geq 0, \quad \forall \tau \in \mathbb{R}_{+}^{n} .
\end{array}\right.
$$

Proof. Let

$$
\widetilde{V}_{j}(\cdot)=\bar{V}_{j}(\cdot)+\operatorname{ind}_{\bar{B}_{\mathbb{R}^{n}(0, K)}}(\cdot), \quad K>\delta+m, \quad j=1, \ldots, m,
$$

where $\delta$ is the constant from $\left(H_{7}\right)$. We claim that the assumptions of Theorem 3.1 are satisfied for the system

$$
\left(\widetilde{V}_{j}(\cdot), \phi_{j}(\cdot)+\varepsilon\|\cdot\|, \Phi_{+}(\cdot)+\varepsilon m\|\cdot\|\right), \quad 0<\varepsilon \leq 1 .
$$

Indeed, the function $\Phi_{+}(\cdot)+\varepsilon m\|\cdot\|\left(\right.$ where $\left.\Phi_{+}=\Phi+\operatorname{ind}_{\mathbb{R}_{+}^{n}}\right)$ is convex, proper, l.s.c. and positive homogeneous of degree 1 for $0<\varepsilon \leq 1$. Hence there exists a convex, closed subset $W^{\varepsilon}$ of $\mathbb{R}^{n}$ such that $\left(\Phi_{+}(\cdot)+\varepsilon m\|\cdot\|\right)^{\star}=\operatorname{ind}_{W^{\varepsilon}}$. From $\left(H_{7}\right)$ we deduce that $W^{\varepsilon} \cap \mathbb{R}_{+}^{n} \subset \bar{B}_{\mathbb{R}^{n}}(0, \delta+m)$ for $0<\varepsilon \leq 1$. If $x_{j} \in \partial \widetilde{V}_{j}^{\star}(0)$, $j=1, \ldots, m$, then $\left(H_{3}^{0}\right)$ and $\left(H_{3}^{1}\right)$ imply that $\left\|x_{j}\right\|=K, j=1, \ldots, m$. Therefore $\sum_{j=1}^{m} x_{j} \notin \bar{B}_{\mathbb{R}^{n}}(0, \delta+m) \cap \mathbb{R}_{+}^{n}$ and $\sum_{j=1}^{m} x_{j} \notin \partial\left(\Phi_{+}+\varepsilon m\|\cdot\|\right)(0)$, for all $0<\varepsilon \leq 1$. Thus assumption $\left(H_{5}\right)$ holds. The remaining assumptions can be easily verified.

Accordingly, Theorem 3.1 implies that for any $0<\varepsilon \leq 1$ there exist $s^{\varepsilon} \geq 1$ and $\pi^{\varepsilon} \in \mathbb{R}_{+}^{n}, \pi^{\varepsilon} \neq 0, x_{j}^{\varepsilon} \in \mathbb{R}_{+}^{n}, \alpha_{j}^{\varepsilon} \in \mathbb{R}_{+}, j=1, \ldots, m$, such that

$$
\left\{\begin{array}{l}
-\alpha_{j}^{\varepsilon} \pi^{\varepsilon} \in \partial \widetilde{V}_{j}\left(x_{j}^{\varepsilon}\right), \\
\left\langle\pi^{\varepsilon}, x_{j}^{\varepsilon}\right\rangle-s^{\varepsilon}\left(\phi_{j}\left(\pi^{\varepsilon}\right)+\varepsilon\left\|\pi^{\varepsilon}\right\|\right) \in \partial \operatorname{ind}_{\geq 0}\left(\alpha_{j}^{\varepsilon}\right), \\
\Phi(\tau)-\Phi\left(\pi^{\varepsilon}\right)+\varepsilon m\left(\|\tau\|-\left\|\pi^{\varepsilon}\right\|\right) \geq\left\langle\tau-\pi^{\varepsilon}, \sum_{j=1}^{m} x_{j}^{\varepsilon}\right\rangle, \quad \forall \tau \in \mathbb{R}_{+}^{n} .
\end{array}\right.
$$

From the definition of $\widetilde{V}_{j}$ we get $\left\|x_{j}^{\varepsilon}\right\| \leq K$ for all $0<\varepsilon \leq 1$ and $j \in$ $\{1, \ldots, m\}$.

Notice that

$$
\alpha_{j}^{\varepsilon}>0, \quad \forall 0<\varepsilon \leq 1, \forall j \in\{1, \ldots, m\} .
$$

Indeed, suppose that $\alpha_{j_{0}}^{\widehat{\varepsilon}}=0$ for some $0<\widehat{\varepsilon} \leq 1$ and $j_{0} \in\{1, \ldots, m\}$. From $(3.2)_{1},\left(H_{3}^{0}\right),\left(H_{3}^{1}\right)$ we obtain $\left\|x_{j_{0}}^{\widehat{\varepsilon}}\right\|=K$. From $(3.2)_{3}$ we get $\left\|\sum_{j=1}^{m} x_{j}^{\widehat{\varepsilon}}\right\| \leq$ $\delta+m$, which contradicts (3.1). form

Since $\alpha_{j}^{\varepsilon}>0$ for $j=1, \ldots, m$, the condition $(3.2)_{2}$ has the equivalent

$$
\left\langle\pi^{\varepsilon}, x_{j}^{\varepsilon}\right\rangle=s^{\varepsilon}\left(\phi_{j}\left(\pi^{\varepsilon}\right)+\varepsilon\left\|\pi^{\varepsilon}\right\|\right), \quad j=1, \ldots, m .
$$


Summing up these equalities and applying $(3.2)_{3}$ we get

$$
\begin{aligned}
\Phi\left(\pi^{\varepsilon}\right)+\varepsilon m\left\|\pi^{\varepsilon}\right\| & =\left\langle\sum_{j=1}^{m} x_{j}^{\varepsilon}, \pi^{\varepsilon}\right\rangle=s^{\varepsilon} \sum_{j=1}^{m}\left(\phi_{j}\left(\pi^{\varepsilon}\right)+\varepsilon\left\|\pi^{\varepsilon}\right\|\right) \\
& =s^{\varepsilon}\left(\Phi\left(\pi^{\varepsilon}\right)+\varepsilon m\left\|\pi^{\varepsilon}\right\|\right) .
\end{aligned}
$$

Hence $s^{\varepsilon}=1$ for all $0<\varepsilon \leq 1$.

Let $p^{\varepsilon}=\pi^{\varepsilon} /\left\|\pi^{\varepsilon}\right\|$ for $0<\varepsilon \leq 1$. There exist $\left(\varepsilon_{k}\right)_{k \in \mathbb{N}}$ and $p \in \mathbb{R}_{+}^{n}$ with $\|p\|=1$ such that $\varepsilon_{k} \rightarrow 0$ and $p^{\varepsilon_{k}} \rightarrow p$ as $k \rightarrow \infty$.

Moreover, notice that

$$
p \in \operatorname{Int} \mathbb{R}_{+}^{n} .
$$

Indeed, suppose that $p \in \operatorname{Fr} \mathbb{R}_{+}^{n}$. We can assume that $p_{1}=0$. As $\Phi(p)>0$, there exists $j_{0} \in\{1, \ldots, m\}$ such that $\phi_{j_{0}}(p)>0$.

We now prove that $\left(\alpha_{j_{0}}^{\varepsilon_{k}}\left\|\pi_{k}^{\varepsilon}\right\|\right)_{k \in \mathbb{N}}$ is bounded. Suppose to the contrary $\alpha_{j_{0}}^{\varepsilon_{k}}\left\|\pi_{k}^{\varepsilon}\right\| \rightarrow \infty$ as $k \rightarrow \infty$ (passing to a subsequence if necessary). Since $\widetilde{V}_{j_{0}}^{\star}(0) \neq \emptyset$ there exists $c_{j_{0}} \in \mathbb{R}$ such that

$$
\widetilde{V}_{j_{0}}(y) \geq-c_{j_{0}}, \quad \forall y \in \operatorname{Dom} \widetilde{V}_{j_{0}} .
$$

From $(3.2)_{1},(3.2)_{2}$ we get

$$
\phi_{j_{0}}\left(p^{\varepsilon_{k}}\right) \leq \frac{c_{j_{0}}+\widetilde{V}_{j_{0}}(y)}{\alpha_{j_{0}}^{\varepsilon_{k}}\left\|\pi_{k}^{\varepsilon}\right\|}+\|y\|, \quad \forall y \in \operatorname{Dom} \widetilde{V}_{j_{0}} .
$$

Letting $k \rightarrow \infty$ we obtain

$$
0<\phi_{j_{0}}(p) \leq\|y\|, \quad \forall y \in \operatorname{Dom} \widetilde{V}_{j_{0}},
$$

which contradicts the assumption $0 \in \operatorname{cl}\left(\operatorname{Dom} \partial \widetilde{V}_{j_{0}}\right)$.

Hence we can assume that there exist $x_{j_{0}} \in \mathbb{R}_{+}^{n}$ and $\widetilde{\alpha}_{j_{0}} \in \mathbb{R}_{+}$such that $x_{j_{0}}^{\varepsilon_{k}} \rightarrow x_{j_{0}}$ and $\alpha_{j_{0}}^{\varepsilon_{k}}\left\|\pi_{k}^{\varepsilon}\right\| \rightarrow \widetilde{\alpha}_{j_{0}}$ as $k \rightarrow \infty$ (passing to a subsequence if necessary). From positive homogeneity of $\phi_{j_{0}}$ of degree 1 we get the equivalent form of $(3.2)_{1},(3.2)_{2}$ :

$$
-\alpha_{j_{0}}^{\varepsilon_{k}}\left\|\pi_{k}^{\varepsilon}\right\| p^{\varepsilon_{k}} \in \partial \widetilde{V}_{j_{0}}\left(x_{j_{0}}^{\varepsilon_{k}}\right), \quad\left\langle p^{\varepsilon_{k}}, x_{j_{0}}^{\varepsilon_{k}}\right\rangle-\phi_{j_{0}}\left(p^{\varepsilon_{k}}\right)-\varepsilon_{k} \in \partial \operatorname{ind}_{\geq 0}\left(\alpha_{j_{0}}^{\varepsilon_{k}}\left\|\pi_{k}^{\varepsilon}\right\|\right) .
$$

Letting $k \rightarrow \infty$ yields

$$
-\widetilde{\alpha}_{j_{0}} p \in \partial \widetilde{V}_{j_{0}}\left(x_{j_{0}}\right), \quad\left\langle p, x_{j_{0}}\right\rangle-\phi_{j_{0}}(p) \in \partial \operatorname{ind}_{\geq 0}\left(\widetilde{\alpha}_{j_{0}}\right)
$$

Hence

$$
V_{j_{0}}\left(x_{j_{0}}\right)=\min \left\{V_{j_{0}}(x):\langle p, x\rangle \leq \phi_{j_{0}}(p), x \in \mathbb{R}_{+}^{n} \cap B_{\mathbb{R}^{n}}(0, K)\right\} .
$$

From $(3.2)_{3}$ we infer that $x_{j_{0}} \in \bar{B}_{\mathbb{R}^{n}}(0, \delta+m)$. It is easy to check that $\left\|x_{j_{0}}\right\|<K$ and $\widetilde{\alpha}_{j_{0}}>0$.

Using the fact $p \in \operatorname{Int} \mathbb{R}_{+}^{n}$ and assumption $\left(H_{6}\right)$ we get $\phi_{j}(p)>0, j=$ $1, \ldots, m$. Similarly to the above case, there exist $x_{j} \in \mathbb{R}_{+}^{n}$ and $\widetilde{\alpha}_{j} \in \mathbb{R}_{+}$, 
$j=1, \ldots, m$, such that $x_{j}^{\varepsilon_{k}} \rightarrow x_{j}$ and $\alpha_{j}^{\varepsilon_{k}}\left\|\pi_{k}^{\varepsilon}\right\| \rightarrow \widetilde{\alpha}_{j}$ as $k \rightarrow \infty$ (passing to a subsequence if necessary) and

$$
-\widetilde{\alpha}_{j} p \in \partial \widetilde{V}_{j}\left(x_{j}\right), \quad\left\langle p, x_{j}\right\rangle-\phi_{j}(p) \in \partial \operatorname{ind}_{\geq 0}\left(\widetilde{\alpha}_{j}\right), \quad j=1, \ldots, m .
$$

and $\left\|x_{j}\right\|<K$. Hence $\partial \widetilde{V}_{j}\left(x_{j}\right)=\partial \bar{V}_{j}\left(x_{j}\right)$ and

$$
V_{j}\left(x_{j}\right)=\min \left\{V_{j}(x):\langle p, x\rangle \leq \phi_{j}(p), x \in \mathbb{R}_{+}^{n}\right\}, \quad j=1, \ldots, m .
$$

From positive homogeneity of $\Phi$ of degree 1 the condition $(3.2)_{3}$ has the equivalent form

$$
\Phi(\tau)-\Phi\left(p^{\varepsilon_{k}}\right)+\varepsilon_{k} m(\|\tau\|-1) \geq\left\langle\tau-p^{\varepsilon_{k}}, \sum_{j=1}^{m} x_{j}^{\varepsilon_{k}}\right\rangle, \quad \forall \tau \in \mathbb{R}_{+}^{n},
$$

(after substituting $\tau /\left\|\pi_{k}^{\varepsilon}\right\|$ for $\tau$ ). Letting $k \rightarrow \infty$ we get

$$
\Phi(\tau)-\Phi(p) \geq\left\langle\tau-p, \sum_{j=1}^{m} x_{j}\right\rangle, \quad \forall \tau \in \mathbb{R}_{+}^{n} .
$$

Taking into account (3.3) we conclude that $p \in \operatorname{Int} \mathbb{R}_{+}^{n}, x_{j} \in \mathbb{R}_{+}^{n}, j=$ $1, \ldots, m$, are a solution of the problem $(\widehat{P})$.

Theorem 3.2 implies

COROLlary 3.3. There exists a classical competitive equilibrium in the Arrow-Debreu and Arrow-Debreu-McKenzie models under the classical assumptions.

4. Examples. Now we recall the main Theorem 5 from [9] (Theorem 10 of [10]).

TheOREM 4.1. Suppose that for any $j=1, \ldots, m$ the following hypotheses hold:

$\left(H_{1}^{0}\right) \quad 0 \in \operatorname{cl}\left(\operatorname{Dom} \partial \bar{V}_{j}\right)$

$\left(H_{8}\right) \quad 0 \notin \partial \bar{V}_{j}(0)$.

Moreover, for any $j=1, \ldots, m$ assume that one of the following conditions holds:

$\left(H_{9}^{0}\right) \operatorname{Dom} \bar{V}_{j}$ is closed

or

$\left(H_{9}^{1}\right)$ if $x_{j}^{k} \rightarrow x_{j}$ and $p^{k} \rightarrow p$ as $k \rightarrow \infty$ with $\left\|p^{k}\right\|=1$ and $\alpha_{j}^{k}>0$ are such that $-\alpha_{j}^{k} p^{k} \in \partial \bar{V}_{j}\left(x_{j}^{k}\right)$ and $\phi_{j}(p)=0$, then $\liminf _{k \rightarrow \infty} \bar{V}_{j}^{\star}\left(-\alpha_{j}^{k} p^{k}\right)>$ $-\infty$.

Additionally, assume that

$\left(H_{7}\right)$ there exists $\delta>0$ such that $\delta\|\tau\| \geq \Phi(\tau)$ for all $\tau \in \mathbb{R}_{+}^{n}$. 
Then there exist $0<r \leq 1$ and $\left(\pi,\left(x_{j}\right),\left(\alpha_{j}\right)\right)$ with $\pi \in \mathbb{R}_{+}^{n}, \pi \neq 0, x_{j} \in \mathbb{R}_{+}^{n}$ and $\alpha_{j} \in \mathbb{R}_{+} \cup\{\infty\}$ for $j=1, \ldots, m$ such that

$$
(P Q) \quad\left\{\begin{array}{l}
-\alpha_{j} \pi \in \partial \bar{V}_{j}\left(x_{j}\right), \quad\left\langle\pi, x_{j}\right\rangle-\phi_{j}(\pi) \in \partial \operatorname{ind}_{\geq 0}\left(\alpha_{j}\right) \quad \text { if } \alpha_{j} \in \mathbb{R}_{+}, \\
-\pi \in \partial^{\infty} \bar{V}_{j}\left(x_{j}\right), \quad\left\langle\pi, x_{j}\right\rangle=\phi_{j}(\pi)=0 \quad \text { if } \alpha_{j}=\infty, \\
\Phi(\pi)=\left\langle\pi, \frac{1}{r} \sum_{j=1}^{m} x_{j}\right\rangle, \quad \frac{1}{r} \sum_{j=1}^{m} x_{j} \in W,
\end{array}\right.
$$

where $W \subset \mathbb{R}_{+}^{n}$ is such that $\Phi_{+}(\tau)=\sup _{y \in W}\langle\tau, y\rangle$ for $\Phi_{+}=\Phi+\operatorname{ind}_{\mathbb{R}_{+}^{n}}$.

REMARK 4.2. Note that $\left(H_{7}\right)$ implies that $W \cap \mathbb{R}_{+}^{n}$ is bounded. Theorem 4.1 is obtained from [9, Theorem 5] or [10, Theorem 10] by applying those theorems to the functions $\widetilde{V}_{j}:=V_{j}+\operatorname{ind}_{\bar{B}_{\mathbb{R}^{n}}(0, K) \cap \mathbb{R}_{+}^{n}}, j=1, \ldots, m$, with the constant $K>0$ such that $W \cap \mathbb{R}_{+}^{n} \subset B_{\mathbb{R}^{n}}(0, K)$. Thanks to the assumption $W \cap \mathbb{R}_{+}^{n} \subset B_{\mathbb{R}^{n}}(0, K)$, any solution of the modified problem becomes a solution of the initial problem $(P Q)$.

Corollary 4.3. Suppose that the hypotheses of Theorem 4.1 are satisfied. Moreover, assume that one of the following conditions holds:

$\left(H_{10}^{0}\right)$ for each $j=1, \ldots, m$ either $\partial \bar{V}_{j}^{\star}(0)=\emptyset$, or $\langle\tau, y\rangle>\phi_{j}(\tau)$ for all $y \in \partial \bar{V}_{j}^{\star}(0)$ and $\tau \in \mathbb{R}_{+}^{n} \backslash\{0\}$,

or

$\left(H_{10}^{1}\right) \partial \bar{V}_{j}^{\star}(0) \cap \partial \Phi_{+}(0)=\emptyset$ for all $j=1, \ldots, m$, where $\Phi_{+}=\Phi+\operatorname{ind}_{\mathbb{R}_{+}^{n}}$.

Then there exists a solution of the problem $(P Q)$ with $r=1$.

REMARK 4.4. $\left(H_{10}^{0}\right)$ and $\left(H_{10}^{1}\right)$ imply that $\alpha_{j}>0, j=1, \ldots, m$. Hence $\left\langle\pi, x_{j}\right\rangle=\phi_{j}(\pi), j=1, \ldots, m$. Moreover, note that $\left(H_{10}^{0}\right)$ includes the classical assumptions $\left(H_{3}^{0}\right),\left(H_{3}^{1}\right)$.

Corollary 4.5. Suppose that the hypotheses of Theorem 4.1 hold and $\left(H_{11}\right)$ for all $j=1, \ldots, m$, all $\tau \in \operatorname{Fr} \mathbb{R}_{+}^{n} \backslash\{0\}$ and all $z_{j} \in \mathbb{R}_{+}^{n}$ with $\left\langle\tau, z_{j}\right\rangle \leq$ $\phi_{j}(\tau)$, there exists $s_{j} \in \mathbb{R}_{+}^{n} \backslash\{0\}$ with $\left\langle s_{j}, \tau\right\rangle=0$ such that $V_{j}\left(z_{j}+s_{j}\right)$ $<V_{j}\left(z_{j}\right)$.

Then there exists a solution of the problem $(P Q)$ with $\pi \in \operatorname{Int} \mathbb{R}_{+}^{n}$.

REMARK 4.6. The proofs of Corollaries 4.3 and 4.5 are similar to the proof of Theorem 3.2. Hence we can also prove the existence of the competitive equilibrium under the classical assumptions using Theorem 4.1.

COROLlary 4.7. Suppose that the hypotheses of Theorem 4.1 are satisfied. Moreover, assume that there exists $\gamma>0$ such that for any $j=1, \ldots, m$, $\left(H_{12}\right)$ if $\left\langle p^{k}, x_{j}^{k}\right\rangle \rightarrow 0$ as $k \rightarrow \infty$ with $\left\|p^{k}\right\|=1$ then $\liminf _{k \rightarrow \infty} \phi_{j}\left(p^{k}\right) \geq \gamma$. Then there exists a solution of the problem $(P Q)$ with $\alpha_{j}<\infty, j=1, \ldots, m$. 
Now we consider three markets with two traders and commodities.

4.1. The equilibrium price with positive coordinates. Consider the following problem: Find $r \in(0,1]$ and $\bar{\pi}=\left(\pi_{1}, \pi_{2}\right) \in \mathbb{R}_{+}^{2}, \bar{\pi} \neq(0,0), \bar{x}_{j}=$ $\left(x_{j}, y_{j}\right) \in \mathbb{R}_{+}^{2}, \alpha_{j} \in \mathbb{R}_{+} \cup\{\infty\}, j=1,2$, such that

$$
\left\{\begin{array}{l}
-\alpha_{j} \bar{\pi} \in \partial \widetilde{V}_{j}\left(\bar{x}_{j}\right), \quad\left\langle\bar{\pi}, \bar{x}_{j}\right\rangle-\left\langle\bar{\pi}, \omega_{j}\right\rangle \in \partial \text { ind }_{\geq 0}\left(\alpha_{j}\right), \quad \text { if } \alpha_{j} \in \mathbb{R}_{+} \\
-\bar{\pi} \in \partial^{\infty} \widetilde{V}_{j}\left(\bar{x}_{j}\right), \quad\left\langle\bar{\pi}, \bar{x}_{j}\right\rangle=\left\langle\bar{\pi}, \omega_{j}\right\rangle=0, \quad \text { if } \alpha_{j}=\infty \\
\left\langle\bar{\pi}, \bar{x}_{1}+\bar{x}_{2}\right\rangle=r\left\langle\bar{\pi}, \omega_{1}+\omega_{2}\right\rangle, \quad \bar{x}_{1}+\bar{x}_{2} \leq r\left(\omega_{1}+\omega_{2}\right)
\end{array}\right.
$$

where the functions $V_{1}, V_{2}: \mathbb{R}^{2} \rightarrow \mathbb{R} \cup\{\infty\}$ take the form

$$
\begin{aligned}
V_{j}(x, y) & =(x-1)^{2}+(y-1)^{2}, \\
\widetilde{V}_{j}(x, y) & :=V_{j}(x, y)+\operatorname{ind}_{\mathbb{R}_{+}^{2} \cap \bar{B}((0,0), 3)}(x, y), \quad j=1,2,
\end{aligned}
$$

and $\omega_{1}=(2,1 / 2), \omega_{2}=(0,5 / 4)$.

Note that $W=(-\infty, 2] \times(-\infty, 7 / 4], \operatorname{Dom} \widetilde{V}_{j}=\mathbb{R}_{+}^{2},(0,0) \notin \partial \bar{V}_{j}(0,0)$, $j=1,2$. The problem (4.1) satisfies all the assumptions of Theorem 4.1, hence it has a solution. Consider the following cases:

CASE 1: $\pi_{1}>0, \pi_{2}=0$. In this case $\left\langle\left(\pi_{1}, 0\right), \omega_{1}\right\rangle>0$ and $\left\langle\left(\pi_{1}, 0\right), \omega_{2}\right\rangle=0$. This implies that $\alpha_{1}<\infty$. Therefore $\left(x_{1}, y_{1}\right)=(1,1)$ and $\alpha_{1}=0$.

If $\alpha_{2}=\infty$, then $\left(x_{2}, y_{2}\right)=\left(0, y_{2}\right), y_{2} \in[0,3]$.

If $\alpha_{2}<\infty$, then $\left(x_{2}, y_{2}\right)=(0,1)$ and $\alpha_{2}>0$ with $\alpha_{2} \pi_{1} \geq 2$.

Moreover, the following conditions must hold:

$$
x_{1}+x_{2}=2 r, \quad y_{1}+y_{2} \leq \frac{7}{4} r, \quad 0<r \leq 1,
$$

which is impossible.

CASE 2: $\pi_{1}=0, \pi_{2}>0$. Then $\left\langle\left(0, \pi_{2}\right), \omega_{1}\right\rangle>0$ and $\left\langle\left(0, \pi_{2}\right), \omega_{2}\right\rangle>0$. From this we infer $\alpha_{1}, \alpha_{2}<\infty$. Then $\left(x_{1}, y_{1}\right)=(1,1 / 2), \alpha_{1}=1 / \pi_{2}$, $\left(x_{2}, y_{2}\right)=(1,1), \alpha_{2}=0$, which contradicts the conditions: $x_{1}+x_{2} \leq 2 r$, $y_{1}+y_{2}=\frac{7}{4} r, 0<r \leq 1$.

CASE 3: $\pi_{1}>0, \pi_{2}>0$. In this case $\left\langle\left(\pi_{1}, \pi_{2}\right), \omega_{1}\right\rangle>0$ and $\left\langle\left(\pi_{1}, \pi_{2}\right), \omega_{2}\right\rangle$ $>0$. We get $\alpha_{1}, \alpha_{2}<\infty$. Therefore

$$
\begin{aligned}
& \left(x_{1}, y_{1}\right)= \begin{cases}(1,1) & \text { if } \pi_{2} \leq 2 \pi_{1}, \\
\left(1+\frac{\pi_{1}\left(2 \pi_{1}-\pi_{2}\right)}{2\left(\pi_{1}^{2}+\pi_{2}^{2}\right)}, 1+\frac{\pi_{2}\left(2 \pi_{1}-\pi_{2}\right)}{2\left(\pi_{1}^{2}+\pi_{2}^{2}\right)}\right) & \text { if } \pi_{2}>2 \pi_{1},\end{cases} \\
& \left(x_{2}, y_{2}\right)= \begin{cases}(1,1) & \text { if } \pi_{2} \geq 4 \pi_{1}, \\
\left(1+\frac{\pi_{1}\left(\pi_{2}-4 \pi_{1}\right)}{4\left(\pi_{1}^{2}+\pi_{2}^{2}\right)}, 1+\frac{\pi_{2}\left(\pi_{2}-4 \pi_{1}\right)}{4\left(\pi_{1}^{2}+\pi_{2}^{2}\right)}\right) & \text { if } \pi_{2}<4 \pi_{1} .\end{cases}
\end{aligned}
$$

Moreover, the following conditions must hold:

$$
x_{1}+x_{2}=2 r, \quad y_{1}+y_{2}=\frac{7}{4} r, \quad 0<r \leq 1 .
$$


Therefore

$$
7\left(x_{1}+x_{2}\right)=8\left(y_{1}+y_{2}\right) .
$$

Consider the following subcases:

- $\pi_{2} \leq 2 \pi_{1}$. Then from (4.2) we get the equation $16 \pi_{2}^{2}-39 \pi_{1} \pi_{2}+36 \pi_{1}^{2}=0$, which does not have a solution.

- $2 \pi_{1}<\pi_{2}<4 \pi_{1}$. Using (4.2) we get the equation $8 \pi_{1}^{2}+7 \pi_{1} \pi_{2}=0$, which does not have a solution for $\pi_{1}>0, \pi_{2}>0$.

- $\pi_{2} \geq 4 \pi_{1}$. Then from $(4.2)$ we get $4 \pi_{2}^{2}-23 \pi_{1} \pi_{2}+10 \pi_{1}^{2}=0$. Hence

$$
\pi_{2}=\frac{23-3 \sqrt{41}}{8} \pi_{1} \approx 0.47 \pi_{1}<4 \pi_{1} \vee \pi_{2}=\frac{23+3 \sqrt{41}}{8} \pi_{1} \approx 5.27 \pi_{1}>4 \pi_{1}
$$

Finally, we have shown that the system

$$
\begin{gathered}
\bar{\pi}=\left(\pi_{1}, \frac{23+3 \sqrt{41}}{8} \pi_{1}\right), \pi_{1}>0, \quad r=\frac{129-3 \sqrt{41}}{113} \approx 0.97, \\
\bar{x}_{1}=\left(\frac{145-6 \sqrt{41}}{113}, \frac{451-21 \sqrt{41}}{452}\right), \quad \bar{x}_{2}=(1,1)
\end{gathered}
$$

is a solution of the problem: Find $r \in(0,1], \bar{\pi}=\left(\pi_{1}, \pi_{2}\right) \in \mathbb{R}_{+}^{2}, \bar{\pi} \neq(0,0)$, and $\bar{x}_{j}=\left(x_{j}, y_{j}\right) \in \mathbb{R}_{+}^{2}, j=1,2$, such that

$$
\left\{\begin{array}{l}
V_{1}\left(x_{1}, y_{1}\right)=\min \left\{V_{1}(x, y): \pi_{1} x+\pi_{2} y \leq 2 \pi_{1}+\frac{1}{2} \pi_{2}, x, y \geq 0\right\} \\
V_{2}\left(x_{2}, y_{2}\right)=\min \left\{V_{2}(x, y): \pi_{1} x+\pi_{2} y \leq \frac{5}{4} \pi_{2}, x, y \geq 0\right\} \\
\pi_{1}\left(x_{1}+x_{2}\right)+\pi_{2}\left(y_{1}+y_{2}\right)=r\left(2 \pi_{1}+\frac{7}{4} \pi_{2}\right), x_{1}+x_{2} \leq 2 r, y_{1}+y_{2} \leq \frac{7}{4} r .
\end{array}\right.
$$

Note that the price vector is uniquely determined among all normalized prices, i.e. elements of the simplex $S_{2}:=\left\{q=\left(q_{1}, q_{2}\right) \in \mathbb{R}_{+}^{2}: q_{1}+q_{2}=1\right\}$.

In the above example, there are price vectors for which the budget sets of two traders include the global maximizers of their utility functions. However, at equilibrium understood in the sense of the variational inequality $(P Q)_{3}$, there exists exactly one normalized price vector for which the traders cannot both attain their global maximum on the corresponding budget sets. Moreover, the above unique solution is not a Walrasian equilibrium. This is a consequence of the fact that $\bar{x}_{2}$ globally maximizes the utility functions of the second trader $-V_{2}$ in the interior of his budget set. Finally, what is worth mentioning, the prices of the two commodities are positive and therefore they are attractive from the point of view of pure exchange economy.

4.2. Walrasian equilibrium. Assume that functions $V_{1}, \widetilde{V}_{1}: \mathbb{R}^{2} \rightarrow \mathbb{R}$ $\cup\{\infty\}$ take the form

$V_{1}(x, y):=(x-1)^{2}+(y-1)^{2}, \quad \widetilde{V}_{1}(x, y):=V_{1}(x, y)+\operatorname{ind}_{\mathbb{R}_{+}^{2} \cap \bar{B}((0,0), 4)}(x, y)$, 
functions $V_{2}: \mathbb{R}_{+}^{2} \rightarrow \mathbb{R}, \widetilde{V}_{2}: \mathbb{R}^{2} \rightarrow \mathbb{R} \cup\{\infty\}$ are given by

$$
V_{2}(x, y):=-\sqrt{x y}, \quad \widetilde{V}_{2}(x, y):= \begin{cases}-\sqrt{x y} & \text { if }(x, y) \in \mathbb{R}_{+}^{2} \cap \bar{B}((0,0), 4), \\ \infty & \text { otherwise, }\end{cases}
$$

and $\omega_{1}=(1,0), \omega_{2}=(0,3)$. Consider the following problem: Find $r \in(0,1]$ and $\bar{\pi}=\left(\pi_{1}, \pi_{2}\right) \in \mathbb{R}_{+}^{2}, \bar{\pi} \neq(0,0), \bar{x}_{j}=\left(x_{j}, y_{j}\right) \in \mathbb{R}_{+}^{2}, \alpha_{j} \in \mathbb{R}_{+} \cup\{\infty\}$, $j=1,2$, such that

$$
\left\{\begin{array}{l}
-\alpha_{j} \bar{\pi} \in \partial \widetilde{V}_{j}\left(\bar{x}_{j}\right), \quad\left\langle\bar{\pi}, \bar{x}_{j}\right\rangle-\left\langle\bar{\pi}, \omega_{j}\right\rangle \in \partial \operatorname{ind}_{\geq 0}\left(\alpha_{j}\right), \quad \text { if } \alpha_{j} \in \mathbb{R}_{+}, \\
-\bar{\pi} \in \partial^{\infty} \widetilde{V}_{j}\left(\bar{x}_{j}\right), \quad\left\langle\bar{\pi}, \bar{x}_{j}\right\rangle=\left\langle\bar{\pi}, \omega_{j}\right\rangle=0, \quad \text { if } \alpha_{j}=\infty \\
\left\langle\bar{\pi}, \bar{x}_{1}+\bar{x}_{2}\right\rangle=r\left\langle\bar{\pi}, \omega_{1}+\omega_{2}\right\rangle, \quad \bar{x}_{1}+\bar{x}_{2} \leq r\left(\omega_{1}+\omega_{2}\right) .
\end{array}\right.
$$

Note that $W=(-\infty, 1] \times(-\infty, 3]$ and $\operatorname{Dom} \widetilde{V}_{j}=\mathbb{R}_{+}^{2} \cap \bar{B}_{\mathbb{R}^{2}}((0,0), 4)$ with $(0,0) \notin \partial \widetilde{V}_{j}(0,0), j=1,2$. From Theorem 4.1 we infer that the problem (4.3) has a solution.

Consider the following cases:

CASE 1: $\pi_{1}=0, \pi_{2}>0$. We have $\left\langle\left(0, \pi_{2}\right), \omega_{1}\right\rangle=0$ and $\left\langle\left(0, \pi_{2}\right), \omega_{2}\right\rangle>0$, which implies $\alpha_{2}<\infty$. Therefore $\left(x_{2}, y_{2}\right)=(\sqrt{8}, \sqrt{8})$ and $\alpha_{2}=0$. This contradicts the conditions: $x_{1}+x_{2} \leq r, y_{1}+y_{2}=3 r, 0<r \leq 1, x_{1} \geq 0$.

CASE 2. $\pi_{1}>0, \pi_{2}>0$. Now $\left\langle\left(\pi_{1}, \pi_{2}\right), \omega_{1}\right\rangle>0$ and $\left\langle\left(\pi_{1}, \pi_{2}\right), \omega_{2}\right\rangle>0$. This implies that $\alpha_{1}, \alpha_{2}<\infty$. Hence

$$
\begin{aligned}
& \left(x_{1}, y_{1}\right)=\left(1-\frac{\pi_{1} \pi_{2}}{\pi_{2}^{1}+\pi_{2}^{2}}, \frac{\pi_{1}^{2}}{\pi_{1}^{2}+\pi_{2}^{2}}\right) \\
& \left(x_{2}, y_{2}\right)= \begin{cases}\left(\frac{3}{2} \frac{\pi_{2}}{\pi_{1}}, \frac{3}{2}\right) & \text { if } 0<\pi_{2} \leq \frac{\sqrt{55}}{3} \pi_{1}, \\
\left(\widetilde{x}_{2},-\frac{\pi_{1}}{\pi_{2}} \widetilde{x}_{2}+3\right) & \text { if } \frac{\sqrt{55}}{3} \pi_{1}<\pi_{2} \leq(3 \sqrt{8}+8) \pi_{1}, \\
(\sqrt{8}, \sqrt{8}) & \text { if } \pi_{2} \geq(3 \sqrt{8}+8) \pi_{1},\end{cases}
\end{aligned}
$$

where

$$
\widetilde{x}_{2}=\frac{\pi_{2}\left(3 \pi_{1}+\sqrt{16 \pi_{1}^{2}+7 \pi_{2}^{2}}\right)}{\pi_{1}^{2}+\pi_{2}^{2}}, \quad \widetilde{x}_{2} \geq \sqrt{8} .
$$

Moreover, the following conditions must hold:

$$
x_{1}+x_{2}=r, \quad y_{1}+y_{2}=3 r, \quad 0<r \leq 1 .
$$

Analysis similar to that in Case 1 leads to a contradiction if $\pi_{2}>\frac{\sqrt{55}}{3} \pi_{1}$. For $0<\pi_{2} \leq \frac{\sqrt{55}}{3} \pi_{1}$ the equation

$$
3\left(x_{1}+x_{2}\right)=y_{1}+y_{2}
$$

leads to $\pi_{1}^{2}+3 \pi_{2}^{2}=0$, which does not have a solution in the set $\pi_{1}, \pi_{2}>0$. 
CASE 3: $\pi_{1}>0, \pi_{2}=0$. Then $\left\langle\left(\pi_{1}, 0\right), \omega_{1}\right\rangle>0$ and $\left\langle\left(\pi_{1}, 0\right), \omega_{2}\right\rangle=0$. Hence $\alpha_{1}<\infty$ and $\alpha_{2}=\infty$, because Dom $\partial V_{2}=\operatorname{Int} \mathbb{R}_{+}^{2}$. Therefore $\left(x_{1}, y_{1}\right)$ $=(1,1), \alpha_{1}=0$ and $\left(x_{2}, y_{2}\right)=\left(0, y_{2}\right), y_{2} \in[0,4]$. Moreover, the following conditions must hold:

$$
x_{1}+x_{2}=r, \quad y_{1}+y_{2} \leq 3 r, \quad 0<r \leq 1,
$$

hence $r=1, y_{2} \in[0,2]$.

Therefore the following system is a solution of the problem (4.3):

$$
\begin{gathered}
\bar{\pi}=\left(\pi_{1}, 0\right), \pi_{1}>0, \quad r=1, \quad \alpha_{1}=0, \quad \alpha_{2}=\infty, \\
\bar{x}_{1}=\left(x_{1}, y_{1}\right)=(1,1), \quad \bar{x}_{2}=\left(x_{2}, y_{2}\right)=\left(0, y_{2}\right), y_{2} \in[0,2] .
\end{gathered}
$$

Finally, the system

$$
\begin{array}{ll}
\bar{\pi}=\left(\pi_{1}, 0\right), \pi_{1}>0, & \bar{x}_{1}=\left(x_{1}, y_{1}\right)=(1,1), \\
\bar{x}_{2}=\left(x_{2}, y_{2}\right)=\left(0, y_{2}\right), y_{2} \in[0,2],
\end{array}
$$

is a solution of the problem: Find $\bar{\pi} \in \mathbb{R}_{+}^{2}, \bar{\pi} \neq(0,0)$, and $\bar{x}_{j}=\left(x_{j}, y_{j}\right) \in \mathbb{R}_{+}^{2}$, $j=1,2$, such that

$$
\left\{\begin{array}{l}
V_{1}\left(x_{1}, y_{1}\right)=\min \left\{V_{1}(x, y): \pi_{1} x+\pi_{2} y \leq \pi_{1}, x, y \geq 0\right\}, \\
V_{2}\left(x_{2}, y_{2}\right)=\min \left\{V_{2}(x, y): \pi_{1} x+\pi_{2} y \leq 3 \pi_{2}, x, y \geq 0\right\}, \\
\pi_{1}\left(x_{1}+x_{2}\right)+\pi_{2}\left(y_{1}+y_{2}\right)=\pi_{1}+3 \pi_{2}, x_{1}+x_{2} \leq 1, y_{1}+y_{2} \leq 3 .
\end{array}\right.
$$

In this example the preferences of the two traders are represented by different utility functions. The first utility function attains its global maximum on the budget set corresponding to a nonzero price vector of the form $\left(\pi_{1}, 0\right)$, $\pi_{1}>0$. The second function is of the Cobb-Douglas type. For the given initial endowments $\omega_{1}$ and $\omega_{2}$ we get a Walrasian equilibrium with a uniquely determined normalized price vector whose second coordinate is 0 . This means that in such economy the second commodity is not attractive. Moreover, the exchange is useless for the second trader because his budget vanishes.

4.3. The equilibrium price with one coordinate zero. Consider the following problem: Find $r \in(0,1]$ and $\bar{\pi}=\left(\pi_{1}, \pi_{2}\right) \in \mathbb{R}_{+}^{2}, \bar{\pi} \neq(0,0), \bar{x}_{j}=$ $\left(x_{j}, y_{j}\right) \in \mathbb{R}_{+}^{2}, \alpha_{j} \in \mathbb{R}_{+} \cup\{\infty\}, j=1,2$, such that

$$
\left\{\begin{array}{l}
-\alpha_{j} \bar{\pi} \in \partial \widetilde{V}_{j}\left(\bar{x}_{j}\right), \quad\left\langle\bar{\pi}, \bar{x}_{j}\right\rangle-\left\langle\bar{\pi}, \omega_{j}\right\rangle \in \partial \operatorname{ind}_{\geq 0}\left(\alpha_{j}\right), \quad \text { if } \alpha_{j} \in \mathbb{R}_{+}, \\
-\bar{\pi} \in \partial^{\infty} \widetilde{V}_{j}\left(\bar{x}_{j}\right), \quad\left\langle\bar{\pi}, \bar{x}_{j}\right\rangle=\left\langle\bar{\pi}, \omega_{j}\right\rangle=0, \quad \text { if } \alpha_{j}=\infty \\
\left\langle\bar{\pi}, \bar{x}_{1}+\bar{x}_{2}\right\rangle=r\left\langle\bar{\pi}, \omega_{1}+\omega_{2}\right\rangle, \quad \bar{x}_{1}+\bar{x}_{2} \leq r\left(\omega_{1}+\omega_{2}\right)
\end{array}\right.
$$

where the functions $V_{1}, V_{2}, \widetilde{V}_{1}, \widetilde{V}_{2}: \mathbb{R}^{2} \rightarrow \mathbb{R} \cup\{\infty\}$ take the form

$$
\begin{aligned}
& V_{j}(x, y):=(x-1)^{2}+(y-1)^{2}, \\
& \widetilde{V}_{j}(x, y):=V_{j}(x, y)+\operatorname{ind}_{\mathbb{R}_{+}^{2} \cap \bar{B}((0,0), 4)}(x, y), \quad j=1,2,
\end{aligned}
$$


and $\omega_{1}=(3 / 2,0), \omega_{2}=(0,3)$. As in Example 4.1, we know that the problem (4.4) has a solution. Consider the following cases:

CASE 1: $\pi_{1}=0, \pi_{2}>0$. Then $\left\langle\left(0, \pi_{2}\right), \omega_{1}\right\rangle=0$ and $\left\langle\left(0, \pi_{2}\right), \omega_{2}\right\rangle>0$. Hence $\alpha_{2}<\infty$. Therefore $\left(x_{2}, y_{2}\right)=(1,1)$ and $\alpha_{2}=0$.

If $\alpha_{1}=\infty$, then $\left(x_{1}, y_{1}\right)=\left(x_{1}, 0\right)$ with $x_{1} \in[0,4]$.

If $\alpha_{1}<\infty$, then $\left(x_{1}, y_{1}\right)=(1,0)$ and $\alpha_{1} \pi_{2} \geq 2$.

Moreover, the following conditions must hold:

$$
x_{1}+x_{2} \leq \frac{3}{2} r, \quad y_{1}+y_{2}=3 r, \quad 0<r \leq 1,
$$

which is impossible.

CASE $2: \pi_{1}>0, \pi_{2}>0$. Now we have $\left\langle\left(\pi_{1}, \pi_{2}\right), \omega_{1}\right\rangle>0$ and $\left\langle\left(\pi_{1}, \pi_{2}\right), \omega_{2}\right\rangle$ $>0$. This implies that $\alpha_{1}, \alpha_{2}<\infty$. Hence

$$
\begin{aligned}
& \left(x_{1}, y_{1}\right)= \begin{cases}(1,1) & \text { if } \pi_{2} \leq \frac{1}{2} \pi_{1}, \\
\left(1+\frac{\pi_{1}\left(\pi_{1}-2 \pi_{2}\right)}{2\left(\pi_{1}^{2}+\pi_{2}^{2}\right)}, 1+\frac{\pi_{2}\left(\pi_{1}-2 \pi_{2}\right)}{2\left(\pi_{1}^{2}+\pi_{2}^{2}\right)}\right) & \text { if } \pi_{2}>\frac{1}{2} \pi_{1},\end{cases} \\
& \left(x_{2}, y_{2}\right)= \begin{cases}\left(1+\frac{\pi_{1}\left(2 \pi_{2}-\pi_{1}\right)}{\pi_{1}^{2}+\pi_{2}^{2}}, 1+\frac{\pi_{2}\left(2 \pi_{2}-\pi_{1}\right)}{\pi_{1}^{2}+\pi_{2}^{2}}\right) & \text { if } \pi_{2}<\frac{1}{2} \pi_{1}, \\
(1,1) & \text { if } \pi_{2} \geq \frac{1}{2} \pi_{1} .\end{cases}
\end{aligned}
$$

Moreover, the following conditions must hold:

$$
x_{1}+x_{2}=\frac{3}{2} r, \quad y_{1}+y_{2}=3 r, \quad 0<r \leq 1 .
$$

Therefore

$$
2\left(x_{1}+x_{2}\right)=y_{1}+y_{2} .
$$

Consider the following subcases:

- $\pi_{2}=\frac{1}{2} \pi_{1}$. Then $x_{1}+x_{2}=2, y_{1}+y_{2}=2$, contrary to (4.5).

- $\pi_{2}<\frac{1}{2} \pi_{1}$. From (4.5) we get the equation $5 \pi_{1} \pi_{2}=0$, which does not have a solution in the set $\pi_{1}>0, \pi_{2}>0$.

- $\pi_{2}>\frac{1}{2} \pi_{1}$. Then (4.5) yields the equation $6 \pi_{2}^{2}-5 \pi_{1} \pi_{2}+6 \pi_{1}^{2}=0$, which does not have a solution.

CASE 3: $\pi_{1}>0, \pi_{2}=0$. Then $\left\langle\left(\pi_{1}, 0\right), \omega_{1}\right\rangle>0$ and $\left\langle\left(\pi_{1}, 0\right), \omega_{2}\right\rangle=0$. Hence $\alpha_{1}<\infty$. Therefore $\left(x_{1}, y_{1}\right)=(1,1)$ and $\alpha_{1}=0$.

If $\alpha_{2}<\infty$, then $\left(x_{2}, y_{2}\right)=(0,1)$ and $\alpha_{2}>0$ with $\alpha_{2} \pi_{1} \geq 2$. The following conditions must hold:

$$
x_{1}+x_{2}=\frac{3}{2} r, \quad y_{1}+y_{2} \leq 3 r, \quad 0<r \leq 1 .
$$

Thus $r=2 / 3$.

If $\alpha_{2}=\infty$, then $\left(x_{2}, y_{2}\right)=\left(0, y_{2}\right)$ with $y_{2} \in \mathbb{R}_{+}$, and the same conditions as above must hold. Hence $r=2 / 3$ and $0 \leq y_{2} \leq 1$. 
Therefore the solutions of the problem (4.4) are

$$
\begin{aligned}
\bar{\pi}=\left(\pi_{1}, 0\right), \pi_{1}>0, \quad r=\frac{2}{3}, & \alpha_{1}=0, \quad \alpha_{2} \pi_{1} \geq 2, \quad \alpha_{2}>0, \\
\bar{x}_{1}=(1,1), & \bar{x}_{2}=(0,1)
\end{aligned}
$$

or

$$
\begin{gathered}
\bar{\pi}=\left(\pi_{1}, 0\right), \pi_{1}>0, \quad r=\frac{2}{3}, \quad \alpha_{1}=0, \quad \alpha_{2}=\infty, \\
\bar{x}_{1}=(1,1), \quad \bar{x}_{2}=\left(0, y_{2}\right), y_{2} \in[0,1] .
\end{gathered}
$$

Moreover, notice that the system

$$
\bar{\pi}=\left(\pi_{1}, 0\right), \pi_{1}>0, \quad r=\frac{2}{3}, \quad \bar{x}_{1}=(1,1), \quad \bar{x}_{2}=(0,1)
$$

is a solution of the problem: Find $r \in(0,1], \bar{\pi}=\left(\pi_{1}, \pi_{2}\right) \in \mathbb{R}_{+}^{2}, \bar{\pi} \neq(0,0)$ and $\bar{x}_{j}=\left(x_{j}, y_{j}\right) \in \mathbb{R}_{+}^{2}, j=1,2$, such that

$$
\left\{\begin{array}{l}
V_{1}\left(x_{1}, y_{1}\right)=\min \left\{V_{1}(x, y): \pi_{1} x+\pi_{2} y \leq \frac{3}{2} \pi_{1}, x, y \geq 0\right\} \\
V_{2}\left(x_{2}, y_{2}\right)=\min \left\{V_{2}(x, y): \pi_{1} x+\pi_{2} y \leq 3 \pi_{2}, x, y \geq 0\right\} \\
\pi_{1}\left(x_{1}+x_{2}\right)+\pi_{2}\left(y_{1}+y_{2}\right)=r\left(\frac{3}{2} \pi_{1}+3 \pi_{2}\right), x_{1}+x_{2} \leq \frac{3}{2} r, y_{1}+y_{2} \leq 3 r .
\end{array}\right.
$$

Note that the utility functions are the same as in Example 4.1, which implies that the budget sets of both traders can include their global maximizers. We get the same form of the solution which is not a Walrasian equilibrium, butwhat distinguishes these results - the second coordinate of the price vector is equal to 0 . For the market it is not important whether the bundle of the second trader maximizes the function $-V_{2}$ on the budget set corresponding to the price vectors $\left(\pi_{1}, 0\right), \pi_{1}>0$, because his initial endowment is zero. Summing up, the initial endowments have strong influence on the derived price vector.

Acknowledgments. The author wishes to express her gratitude to Z. Naniewicz for many stimulating conversations.

\section{References}

[1] C. D. Aliprantis, D. J. Brown and O. Burkinshaw, Existence and Optimality of Competitive Equilibria, Springer, 1989.

[2] K. Arrow and G. Debreu, Existence of an equilibrium for a competitive economy, Econometrica 22 (1954), 264-290.

[3] J.-P. Aubin, Optima and Equilibria, Springer, 1993.

[4] F. H. Clarke, Optimization and Nonsmooth Analysis, Wiley, 1983.

[5] A. Mas-Colell, M. D. Whinston and J. R. Green, Microeconomic Theory, Oxford Univ. Press, 1995.

[6] L. McKenzie, On the existence of general equilibrium for a competitive market, Econometrica 27 (1959), 54-71.

[7] Z. Naniewicz, On some optimization problem related to economic equilibrium, Control Cybernet. 31 (2002), 141-165. 
[8] Z. Naniewicz and M. Nockowska, On variational approach to economic equilibriumtype problem, Ann. Math. Sil. 20 (2006), 57-71.

[9] - , - System of variational inequalities related to economic equilibrium, Control Cybernet. 36 (2007), 889-909.

[10] M. Nockowska, System of variational inequalities in modeling of economic equilibrium problems, $\mathrm{PhD}$ dissertation, Cardinal Stefan Wyszyński Univ., 2006 (in Polish).

[11] R. T. Rockafellar and R. J.-B. Wets, Variational Analysis, Springer, 1998.

[12] H. R. Varian, Intermediate Microeconomics. A Modern Approach, Norton, New York, 1999.

Institute of Mathematics

Technical University of Łódź

Wólczańska 215

90-924 Łódź, Poland

E-mail: magdan@p.lodz.pl

Received on 18.8.2008;

revised version on 6.1.2009 